\title{
Gemcitabine and oxaliplatinum: an effective regimen in patients with refractory and relapsing Hodgkin lymphoma
}

\author{
Antonio Gutierrez ${ }^{1, *}$ \\ Jose Rodriguez ${ }^{1, *}$ \\ Jordi Martinez-Serra' \\ Jordi Gines ${ }^{2}$ \\ Pilar Paredes' \\ Florencia Garcia ${ }^{3}$ \\ Javier Vercher 4 \\ Josep Balanzat ${ }^{4}$ \\ Raquel del Campo ${ }^{5}$ \\ Pilar Galan ${ }^{6}$ \\ Miguel Morey' \\ Antonia Sampol, \\ Andres Novo' \\ Leyre Bento' \\ Lucia García' \\ Joan Bargay ${ }^{5}$ \\ Joan Besalduch ${ }^{1,7}$
}

'Service of Hematology, ${ }^{2}$ Service of Pharmacy, ${ }^{3}$ Service of Oncology, Son Espases University Hospital, Palma de Mallorca, Spain; ${ }^{4}$ Service of Hematology, Can Misses Hospital, Ibiza, Spain; ${ }^{5}$ Service of Hematology, Son Llatzer Hospital, Palma, Spain; ${ }^{6}$ Service of Hematology, Mateu Orfila Hospital, Menorca, Spain; ${ }^{7}$ Service of Hematology, Policlínica Miramar, Instituto de Investigación Sanitaria de Palma (IdISPa), Palma, Spain

*These authors contributed equally to this work

Correspondence: Antonio Gutierrez Service of Hematology, Son Espases University Hospital, Carretera de Valldemossa, 7907010 Palma de Mallorca, Spain

Tel +34 87I 205000 , ext 65II8

Fax +34 87I 909717

Email antoniom.gutierrez@ssib.es
This article was published in the following Dove Press journal:

OncoTargets and Therapy

13 November 2014

Number of times this article has been viewed

Background: Most Hodgkin lymphomas (HL) can be cured with current strategies. However, one-third of the cases do not respond or relapse and need salvage regimens. We report the results of a retrospective study using the gemcitabine and oxaliplatinum (GemOx) regimen.

Methods: Patients who relapsed or failed to achieve complete response were eligible and received GemOx salvage therapy. To avoid selection bias and thus to overcome the retrospective nature of the study, all treated patients were included from the pharmacy database.

Results: Between 2003-2013, 24 HL patients - relapsing (number [n]=12) or refractory $(n=12)$ - were included, receiving a total of 26 induction treatments with GemOx. Mean previous regimens were 2.38 (42\% relapsing after autologous transplantation). Median follow-up was 37 months, and $71 \%$ responded ( $38 \%$ of patients achieved complete response). The factors related to better progression-free survival were: B symptoms; response to GemOx; and consolidation with stem cell transplantation. Grades 1 and 2 neurological toxicity was present in $17 \%$ of patients. Hematological toxicity was common, with grades 3 and 4 neutropenia (25\%) and thrombocytopenia (34\%) observed. Progression-free survival was better in patients consolidated with stem cell transplantation. The peripheral blood stem cell collection after GemOx was successful for all candidates.

Conclusion: 1) The GemOx regimen is effective in relapsed or refractory HL with manageable toxicity. 2) No mobilization failures were observed. 3) Consolidation after response is needed. 4) Its efficacy and favorable toxicity profile might make multiple administrations possible in several recurrences in HL.

Keywords: gemcitabine, oxaliplatinum, GemOx, Hodgkin lymphoma, treatment

\section{Introduction}

Hodgkin lymphoma (HL) represents $10 \%-15 \%$ of all types of lymphomas. ${ }^{1}$ At present, more than $70 \%$ of patients can be cured with the current strategies based on chemotherapy, with or without radiotherapy. However, one-third of the cases finally relapse and need salvage regimens that are usually consolidated with high-dose chemotherapy and autologous stem cell transplantation (ASCT). ${ }^{2}$

The patients that relapse after ASCT pose a great challenge as there is no standard strategy, although new drugs such as brentuximab vedotin have been approved in this setting. ${ }^{3}$ Furthermore, some cases have a pattern of relapses and remissions, which defines a chronic evolution. There are many salvage regimens (intensive nonmyeloablative regimens, such as mini-BEAM (carmustine, etoposide, cytarabine, melphalan), platinum-based regimens, ifosfamide-based and etoposide-based protocols, gemcitabine-based combinations, and new anti-CD30-based immunoconjugates), but there are no prospective randomized trials comparing one regimen with another. 
These regimens obtain overall response rates (ORR) between $70 \%-88 \%$ with complete response (CR) rates ranging from $19 \%-54 \%$, depending on the type of patients and disease (Table 1). However, most regimens are quite toxic and difficult to administer more than once to the same patient. For this reason, new drugs and combinations are needed to obtain both significant activity and a favorable toxicity profile, especially for patients experiencing multiple relapses. Ideally, these combinations should also not inhibit peripheral blood progenitor cell mobilization, since consolidation with ASCT is important in the first relapse. ${ }^{4}$

We previously reported the clinical activity of the gemcitabine and oxaliplatinum $(\mathrm{GemOx})$ regimen in diffuse large B-cell ${ }^{5}$ and mantle cell ${ }^{6}$ lymphoma with good results in the salvage setting. Combinations with gemcitabine have been tested in HL and are less toxic, and are better mobilizers, with better results noted in terms of progression-free survival (PFS) compared to intensive salvage regimens. ${ }^{7}$ In terms of efficacy and toxicity, the reported results are promising with the oxaliplatin-based regimen of combined ifosfamide, etoposide, and oxaliplatin (IVOx). ${ }^{8}$ Herein, we present our experience with the GemOx regimen in relapsed or refractory HL.

\section{Materials and methods}

\section{Patients and study design}

Between 2003-2012, 24 patients with HL were included in a retrospective study of salvage treatment with GemOx from the following centers in Spain: Son Espases Hospital, Palma de Mallorca; Son Llàtzer Hospital, Palma de Mallorca; Can Misses Hospital, Ibiza; and Mateu Orfila Hospital, Menorca. We obtained approval from the hematology research review board of Son Espases Hospital. We had the following inclusion criteria: patients with a diagnosis with HL, which relapsed or failed to achieve CR after induction treatment; the provision of signed informed consent; and patients who received GemOx salvage therapy. The exclusion criteria were: patients with severe concomitant medical or psychiatric illnesses; HIV-positive; bilirubin levels $>1.5 \mathrm{mg} / \mathrm{dL}$; a cardiac ejection fraction $<50 \%$; creatinine clearance $<30 \mathrm{~mL} /$ minute; a pumonary function test; and diffusing lung capacity $<50 \%$ of the predictive value. The pathologist in each center made the histological diagnosis. We obtained the patients from a pharmacy database to avoid selection bias, and we made sure to include all the patients with HL who had received the GemOx regimen in this 10 -year period with an intention to treat. Primary and secondary endpoints were ORR and PFS, respectively.

A retrospective review of staging and response assessment of every case was made to warrant uniform criteria. The disease stage was evaluated according to the Ann Arbor staging system, and patients were staged according to the standard procedures of physical examination, blood and serum assays, chest X-rays, and a computed tomography scan of the neck, chest, abdomen, and pelvis. Bone marrow aspirates and biopsies were obtained prior to therapy.

Table I Selected salvage regimens in relapsed or refractory $\mathrm{HL}$

\begin{tabular}{|c|c|c|c|c|}
\hline Regimen & Patients & ORR (CR) & $\begin{array}{l}\text { Grade } 3-4 \text { hematologic } \\
\text { toxicity }\end{array}$ & $\begin{array}{l}\text { Grade 3-4 nonhematologic } \\
\text { toxicity }\end{array}$ \\
\hline \multicolumn{5}{|c|}{ Nonmyeloablative intensive regimens } \\
\hline Mini-BEAM 22 & 55 & $84 \%(51 \%)$ & $86 \%$ & $61 \%$ \\
\hline Dexa-BEAM ${ }^{17,23}$ & 144 & $81 \%(27 \%)$ & $100 \%^{23}$ & $49 \%$ \\
\hline \multicolumn{5}{|l|}{ Platinum-based } \\
\hline DHAP 18 & 102 & $88 \%(21 \%)$ & $48 \%$ & $24 \%$ \\
\hline ASHAP $^{24}$ & 56 & $70 \%(34 \%)$ & $100 \%$ & $0 \%$ \\
\hline ESHAP ${ }^{19}$ & 22 & $73 \%(41 \%)$ & $59 \%$ & NS \\
\hline \multicolumn{5}{|l|}{ Ifosfamide-based } \\
\hline MINE $^{25}$ & 100 & $75 \%(35 \%)$ & $92 \%$ & $16 \%$ \\
\hline $\mathrm{ICE}^{20,26}$ & 65 & $88 \%(26 \%)$ & $72 \%^{26}$ & NS \\
\hline $\operatorname{IVOx}^{8}$ & 34 & $76 \%(32 \%)$ & $9 \%$ & NS \\
\hline \multicolumn{5}{|l|}{ Gemcitabine-based } \\
\hline GVD $^{21}$ & 91 & $70 \%(19 \%)$ & $63 \%$ & $8 \%$ \\
\hline IGEV$^{27}$ & 91 & $81 \%(54 \%)$ & $28 \%$ & $5 \%$ \\
\hline \multicolumn{5}{|l|}{ New drugs } \\
\hline Bendamustine $^{16}$ & 36 & $53 \%(33 \%)$ & $34 \%$ & $14 \%$ \\
\hline Brentuximab vedotin 15 & 102 & $73 \%(32 \%)$ & $21 \%$ & $17 \%$ \\
\hline
\end{tabular}

Abbreviations: ORR, overall response rate; CR, complete response; BEAM, carmustine, etoposide, cytarabine, melphalan; DHAP, cisplatin, cytarabine, dexamethasone; ASHAP, adriamycin, cisplatin, cytarabine, methylprednisolone; ESHAP, etoposide, cisplatin, cytarabine, methylprednisolone; NS, not significant; MINE, mesna, ifosfamide, mitoxantrone, etoposide; ICE, ifosfamide, carboplatin, etoposide; IVOx, ifosfamide, etoposide, oxaliplatin; GVD, gemcitabine, vinorelbine, and pegylated liposomal doxorubicin; IGEV, ifosfamide, gemcitabine, vinorelbine. 
Standard variables of the International Prognostic Score ${ }^{9}$ and other variables of known prognostic importance in this type of lymphoma were evaluated. CR was defined as the disappearance of all clinical evidence of lymphoma for a minimum of 4 weeks, with no persisting symptoms related to the disease. Partial response (PR) was defined as a decrease $>50 \%$ in the sum of the products of the two longest diameters of all measurable lesions for at least 4 weeks, while immeasurable lesions had to have decreased by at least $50 \%$. Lesions that increased in size or new lesions could not be considered as PR. Progressive disease was defined as any increase $>25 \%$ in the sum of the diameter of any measurable lesions or the appearance of a new lesion. Patients who did not achieve PR or who had progressive disease within 1 month of therapy were considered refractory patients. Relapsed or refractory patients were confirmed with a biopsy.

Patients who did not achieve CR after the induction regimen, or who were relapsing, were given GemOx. This regimen consisted of gemcitabine $1,000 \mathrm{mg} / \mathrm{m}^{2}$ and oxaliplatin $100 \mathrm{mg} / \mathrm{m}^{2}$ on day 1 . If feasible, treatment was given every 15 days and, if not, every 21 days. No use of prophylactic growth factors was contemplated. No dose reductions were planned relating to prior received therapy.

\section{Response, toxicity assessment, and follow-up criteria}

We used the standard guidelines for evaluations: ${ }^{10}$ physical examination; complete blood counts; serum biochemistry; bone marrow aspiration or biopsy; and radiological studies, as mentioned previously. We used the World Health Organization criteria to assess the toxicity relating to the regimen. We always considered toxic mortality if it was related to the procedure.

\section{Statistical methods}

PFS (time to progression/relapse) and overall survival (OS) (time to death) were measured from the date of GemOx onset, and were estimated according to the Kaplan-Meier method. ${ }^{11}$ We performed the comparisons between those interest variables with the log-rank test. ${ }^{12} \mathrm{~A}$ comparison between categorical variables was made with the chi-square of Fisher's exact test, as appropriate. All reported $P$-values were two-sided, and statistical significance was defined at $P<0.05$.

\section{Results}

\section{Patient characteristics}

From 2003-2013, we included 24 patients with HL in this study. All patients had relapsing (number $[n]=12$ ) or refractory $(n=8)$ disease, or less than a CR after induction $(n=4)$ of therapy. Drugs were administered for compassionate use. The clinical characteristics of the patients at diagnosis and prior to GemOx are reflected in Table 2. The median age was 27 years (14-76 years), and 49\% had an International Prognostic Score higher than 2. Patients received a median of two previous regimens (1-6), and 71\% received more than one regimen before $\mathrm{GemOx}$, with ten patients $(42 \%)$ relapsing after a prior ASCT (seven just before GemOx with a median response duration of 7 months, and three receiving from 2-3 additional lines prior to GemOx, as shown in Table 3). Table 3 shows the previous lines of therapy used before the GemOx salvage treatment. Most patients received GemOx using a 21-day interval $(n=17)$, and only seven patients received GemOx with a 14-day interval.

\section{Response and outcome}

The ORR was 71\%: 38\% of responses were CRs; and 33\% were PRs. Patients relapsing after ASCT $(n=10)$ also had good response rates: ORR of $90 \%$ with $50 \% \mathrm{CR}$. With a median follow-up for living patients of 37 months (3-98 months), the median OS and PFS were 26 months and 14 months, respectively. As of the last follow-up, nine patients (37\%) are still alive, and 15 patients $(62 \%)$ had already died - ten $(42 \%)$ of these were due to disease progression, four (14\%) due to complications from a subsequent allogeneic transplant (graft versus host disease, thrombotic thrombocytopenic purpura, and bleeding), and one (3\%) due to pneumonia. Interestingly, a patient who relapsed after five prior lines of treatment obtained a CR with the GemOx regimen, relapsing once again 31 months after this salvage treatment. This patient was given GemOx again and achieved a new $\mathrm{CR}$ that was maintained

Table 2 Characteristics of patients

\begin{tabular}{ll}
\hline Characteristics at diagnosis & $\begin{array}{l}\text { Number of } \\
\text { patients (\%) }\end{array}$ \\
\hline Median age, years (range) & $27(14-76)$ \\
Sex (male/female) (\%) & $50 \% / 50 \%$ \\
ECOG PS $>2$ & $2(8 \%)$ \\
Ann Arbor stage III-IV & $13(54 \%)$ \\
IPS $>2$ & $11(49 \%)$ \\
B symptoms & $12(50 \%)$ \\
$>$ I extranodal site & $6(25 \%)$ \\
Bulky disease & $6(25 \%)$ \\
Characteristics pre-GemOx & \\
$\quad$ Median previous lines (range) & $2(1-6)$ \\
$>$ I previous line & $17(71 \%)$ \\
Previous ASCT & $10(42 \%)$ \\
\hline
\end{tabular}

Abbreviations: ECOG, Eastern Cooperative Oncology Group; PS, performance status; IPS, International Prognostic Score; GemOx, gemcitabine and oxaliplatinum; ASCT, autologous stem cell transplantation. 
Table 3 Previous treatment lines

\begin{tabular}{|c|c|c|}
\hline Previous lines & $\mathbf{N}$ & Treatment \\
\hline \multirow[t]{2}{*}{1} & 7 & First: ABVD ( $n=6 / 7)$ \\
\hline & & $A B V D+A S C T(n=I / 7)$ \\
\hline \multirow[t]{4}{*}{2} & 8 & First: ABVD \\
\hline & & Second: ESHAP $(n=3 / 8)$ \\
\hline & & ESHAP + ASCT $(n=4 / 8)$ \\
\hline & & $\operatorname{DHAP}(\mathrm{n}=\mathrm{I} / 8)$ \\
\hline \multirow[t]{9}{*}{3} & 6 & First: ABVD \\
\hline & & Second: ABVD $(n=I / 6)$ \\
\hline & & $\operatorname{ESHAP}(n=1 / 6)$ \\
\hline & & $\operatorname{MOPP}(n=I / 6)$ \\
\hline & & $\operatorname{DHAP}(n=2 / 6)$ \\
\hline & & $\operatorname{BEACOPP}(n=I / 6)$ \\
\hline & & Third: IFE $(n=I / 6)$ \\
\hline & & $\operatorname{ESHAP}(n=3 / 6)+\operatorname{ASCT}(n=1 / 6)$ \\
\hline & & RT $(n=2 / 6)+\operatorname{ASCT}(n=I / 5)$ \\
\hline \multirow[t]{8}{*}{4} & 2 & First: ABVD ( $n=I / 2)$ \\
\hline & & $\mathrm{CHOP}(\mathrm{n}=\mathrm{I} / 2)$ \\
\hline & & Second: DHAP + ASCT $(n=1 / 2)$ \\
\hline & & HyperCVAD (n=I/2) \\
\hline & & Third: IFE $(n=I / 2)$ \\
\hline & & $\mathrm{ESHAP}+\mathrm{ASCT}(\mathrm{n}=\mathrm{I} / 2)$ \\
\hline & & Fourth: methotrexate + vincristine $(n=l / 2)$ \\
\hline & & $A B V D(n=I / 2)$ \\
\hline \multirow[t]{7}{*}{6} & 1 & First: ABVD \\
\hline & & Second: MOPP \\
\hline & & Third: ESHAP + ASCT \\
\hline & & Fourth: vinblastine/procarbazine/ \\
\hline & & cyclophosphamide/prednisone \\
\hline & & Fifth: RT \\
\hline & & Sixth: MINE \\
\hline
\end{tabular}

Abbreviations: $\mathrm{N}$, total number;ABVD, adriamycin,bleomycin,vinblastine, dacarbazine; $\mathrm{n}$, sample number; ASCT, autologous stem cell transplantation; ESHAP, etoposide, cisplatin, cytarabine, methylprednisolone; DHAP, cisplatin, cytarabine, dexamethasone; MOPP, nitrogen mustard, vincristine, procarbazine, prednisone; BEACOPP, bleomycin, etoposide, adriamycin, cyclophosphamide, vincristine, procarbazine, and prednisone; IFE, ifosfamide, etoposide; RT, radiotherapy; HyperCVAD, cyclophosphamide, vincristine, adriamycin, dexamethasone; MINE, mesna, ifosfamide, mitoxantrone, etoposide; $\mathrm{CHOP}$, cyclophosphamide, vincristine, adriamycin, prednisone.

for 17 months, followed by a third consecutive GemOx line with a PR that lasted 11 additional months.

The main prognostic factors for HL were assessed to view their impact on survival. Factors related with PFS were: B symptoms; response to GemOx; and consolidation with stem cell transplantation. Obtaining a $\mathrm{CR}$ after GemOx also meant a better OS (Table 4 and Figure 1).

\section{Safety}

A median of four cycles (1-12) of GemOx was administered per patient, and progression for a total of 123 cycles was evaluated in the current study ( $n=24$ patients, with one patient receiving GemOx in three consecutive progressions). Table 5 shows the adverse events associated with GemOx. Neurologic toxicity was present in $17 \%$ of patients; all of them were grade
1 or 2 . Hematological toxicity was also common, including grade 3 or 4 neutropenia in $25 \%$ of patients, and grade 3 or 4 thrombocytopenia in $33 \%$ of patients. Nausea and vomiting occurred in all the patients at grade 2 or lower. No differences in neurological or hematological toxicity were seen in the analysis of toxicity when comparing patients with only one previous line of treatment against those with more than one.

\section{Peripheral blood stem cell collection and hematopoietic stem cell transplantation}

This is a high-risk series, as ten patients $(42 \%)$ had relapsed after a prior ASCT. Another four patients were ineligible due to advanced age or comorbidities. So, only ten patients were potential candidates for ASCT before GemOx. However, five remained refractory, and only five patients were finally considered candidates for ASCT. Peripheral blood stem cell collection after GemOx and granulocyte colony-stimulating factor was successful with all of them. All cases received BEAM as the conditioning regimen. The outcome in these cases was excellent, with median PFS and OS not reached after a median follow-up of 69 months (23-81). At the last follow-up, only one of these five patients $(20 \%)$ had relapsed and died. Five patients receiving GemOx when relapsing after a prior ASCT were consolidated with nonmyeloablative allogeneic stem cell transplantation (three related and two unrelated). Three cases died due to severe posttransplant complications, and two are still alive and disease-free.

\section{Discussion}

Although this is a retrospective series, we included all HL patients treated with GemOx from the pharmacy databases in this 10-year period in an intention-to-treat analysis to avoid selection bias. Our results show an encouraging effect of GemOx on relapsed or refractory HL in a poor-risk population, with $42 \%$ of patients relapsing after ASCT. GemOx also featured a manageable toxicity profile that made multiple administrations of the regimen possible. Even though limited patient numbers have been included in two other reports and mixed with patients treated with other regimens or types of lymphoma, ${ }^{13,14}$ the present manuscript is the first one that specifically investigates the GemOx regimen in HL.

In spite of the multiple chemotherapeutic regimes that have been tested in relapsed or refractory HL, no prospective randomized trial has ever assessed which is the best combination in terms of efficacy or toxicity. Most important traditional salvage regimens in HL include intensive nonmyeloablative regimens such as: mini-BEAM; platinumbased regimens; ifosfamide- and etoposide-based protocols; 
Table 4 Prognostic factors related to OS and PFS

\begin{tabular}{|c|c|c|c|c|c|}
\hline Factor & $\mathbf{N}$ & $\begin{array}{l}\text { Median OS } \\
\text { (months) }(95 \% \mathrm{Cl})\end{array}$ & $P$-value & $\begin{array}{l}\text { Median PFS } \\
\text { (months) }(95 \% \mathrm{Cl})\end{array}$ & $P$-value \\
\hline Age at diagnosis & & & 0.66 & & 0.088 \\
\hline 14-44 years & 18 & $28(0-58)$ & & $31(0-63)$ & \\
\hline$\geq 45$ years & 6 & $19(13-25)$ & & $6(0-16)$ & \\
\hline Sex & & & 0.50 & & 0.74 \\
\hline Male & 12 & $19(0-38)$ & & $3 I(2-6 I)$ & \\
\hline Female & 12 & $28(7-49)$ & & II (4-19) & \\
\hline Number of previous lines & & & 0.40 & & 0.52 \\
\hline I & 7 & 26 (NA) & & NR & \\
\hline$>1$ & 17 & $16(0-43)$ & & $14(2-27)$ & \\
\hline Duration response prior $\mathrm{GemOx}$ & & & 0.85 & & 0.89 \\
\hline $0-12$ months & 21 & $26(12-40)$ & & $17(3-32)$ & \\
\hline$>12$ months & 3 & $28(0-62)$ & & $14(1-27)$ & \\
\hline Status at GemOx & & & 0.34 & & 0.56 \\
\hline PR or relapse & 15 & $28(10-46)$ & & $17(0-40)$ & \\
\hline Refractory & 9 & $19(0-38)$ & & $9(0-19)$ & \\
\hline Ann Arbor stage at diagnosis & & & 0.56 & & $0.08 \mathrm{I}$ \\
\hline I-II & 11 & $16(0-37)$ & & $6(2-10)$ & \\
\hline III-IV & 13 & $34(13-54)$ & & NR & \\
\hline B symptoms at diagnosis & & & 0.10 & & 0.024 \\
\hline No & 12 & $34(0-70)$ & & 31 (NA) & \\
\hline Yes & 12 & $16(6-27)$ & & $6(0-12)$ & \\
\hline Bulky disease at diagnosis & & & 0.78 & & 0.77 \\
\hline Yes & 6 & $28(23-33)$ & & $4(0-15)$ & \\
\hline No & 16 & $16(4-29)$ & & $17(2-33)$ & \\
\hline IPS at diagnosis & & & 0.26 & & 0.22 \\
\hline $0-2$ & 12 & 99 (NA) & & 31 (NA) & \\
\hline $3-7$ & 11 & $26(12-039)$ & & $9(2-16)$ & \\
\hline Response to GemOx & & & 0.064 & & $<0.001$ \\
\hline $\mathrm{CR}$ & 9 & 99 (NA) & & NR & \\
\hline$P R$ & 8 & $26(0-56)$ & & $17(||-24)$ & \\
\hline Refractory & 7 & $13(3-23)$ & & $2(0-5)$ & \\
\hline Response to GemOx & & & 0.043 & & 0.062 \\
\hline $\mathrm{CR}$ & 9 & 99 (NA) & & NR & \\
\hline Less than $\mathrm{CR}$ & 15 & $19(0-38)$ & & II (0-23) & \\
\hline Consolidation with SCT & & & 0.14 & & $<0.001$ \\
\hline Yes & 10 & NR & & NR & \\
\hline No & 14 & $16(7-26)$ & & $6(2-11)$ & \\
\hline Consolidation with type SCT & & & 0.14 & & 0.001 \\
\hline Allo-SCT & 5 & $6(0-35)$ & & NR & \\
\hline ASCT & 5 & NR & & NR & \\
\hline No & 14 & $16(7-26)$ & & $6(2-11)$ & \\
\hline
\end{tabular}

Abbreviations: OS, overall survival; PFS, progression-free survival; N, number; $\mathrm{Cl}$, confidence interval; NA, not available; NR, not reached; GemOx, gemcitabine and oxaliplatinum; PR, partial response; IPS, International Prognostic Score; CR, complete response; SCT, stem cell transplantation; Allo-SCT, allogeneic stem cell transplantation; ASCT, autologous stem cell transplantation.

and gemcitabine-based combinations. ${ }^{4}$ Also, most of them must be administered on an inpatient basis. Recently, new drugs, such as bendamustine and a new antibody-drug conjugate targeting $\mathrm{CD} 30$ brentuximab vedotin, have demonstrated efficacy in relapsed/refractory HL. ${ }^{15,16}$

In terms of efficacy, all the traditional and new salvage protocols reach ORRs of between $70 \%-88 \%$ with CR rates (CRR) from $19 \%-54 \%$, depending on the type of patients and disease included in each report (Table 1). Our results with GemOx (71\% ORR and 38\% CRR) are encouraging, as they have been obtained in a heavily pretreated series of HL (2.38 median prior regimens and $42 \%$ relapses after ASCT). These results compare favorably with most traditional salvage regimens, particularly when toxicity issues are considered ( $46 \%$ grade 3 or 4 hematologic toxicity as the only severe toxicity). ${ }^{8,17-21}$

Furthermore, the GemOx regimen is an easily manageable outpatient regimen. GemOx combines the efficacy 

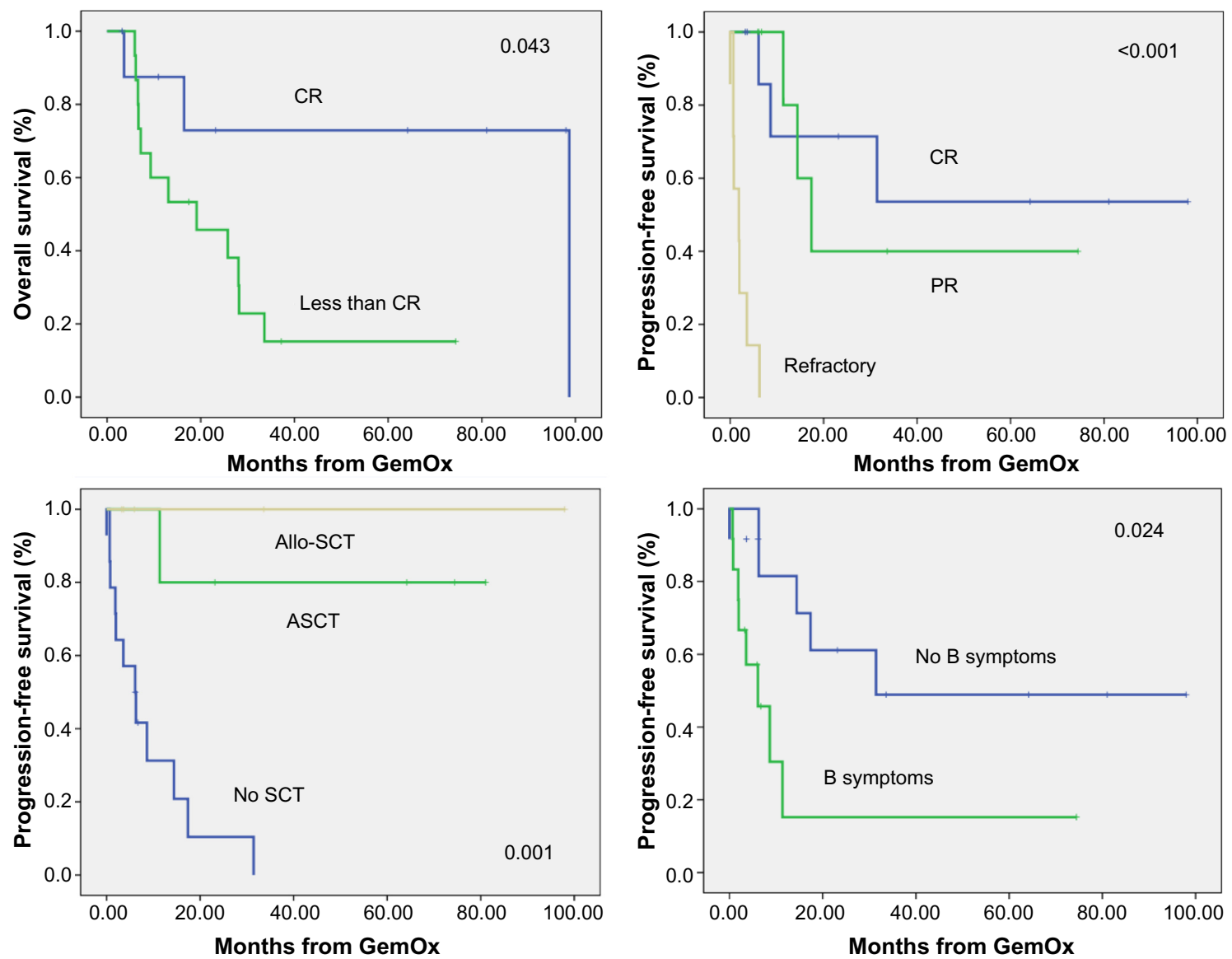

Figure I Prognostic factors influencing OS and progression-free survival.

Abbreviations: CR, complete response; GemOx, gemcitabine and oxaliplatinum; PR, partial response; Allo-SCT, allogenic stem cell transplantation; ASCT, autologous stem cell transplantation; SCT, stem cell transplantation; OS, overall survival.

of gemcitabine and platinum-based regimens with lower toxicity, due to the addition of the less toxic oxaliplatin, which is devoid of the renal and otic toxicity present with cisplatin. We previously reported our experience in both diffuse large B-cell and mantle cell lymphoma in heavily pretreated patients with a high ORR of $43 \%$ and $85 \%$, and grade 3-4 hematological toxicity of $43 \%$ and $35 \%$, respectively. ${ }^{5,6}$ In mantle cell lymphoma, such as in HL, we found that this low-toxicity profile allowed us to administer the treatment consecutively and several times in the same patient. The tolerance and response were good. This may

Table 5 Maximum toxicity

\begin{tabular}{llll}
\hline Toxicities & \multicolumn{2}{l}{$\begin{array}{l}\text { Maximum WHO toxicity grade } \\
\text { (number of patients and \%) }\end{array}$} \\
\cline { 2 - 4 } & I-2 & 3 & $\mathbf{4}$ \\
\hline Neuropathy & $4(17 \%)$ & 0 & 0 \\
Neutropenia & $3(12 \%)$ & $4(17 \%)$ & $2(8 \%)$ \\
Anemia & $7(29 \%)$ & $2(8 \%)$ & $2(8 \%)$ \\
Thrombocytopenia & $6(25 \%)$ & $4(17 \%)$ & $4(17 \%)$ \\
\hline
\end{tabular}

Abbreviation: WHO, World Health Organization. be particularly important in HL where some patients become chronic with multiple relapses and responses. In this series, we present a case that was controlled with three consecutive administrations of GemOx for 5 years, achieving two CRs and one PR.

Since ASCT is important in consolidating response in relapsed or refractory HL, a salvage regimen should ideally not inhibit future peripheral blood stem cell mobilization. Adequate stem cell collection was more likely after gemcitabine-based protocols than after intensive conventional regimens. ${ }^{7}$ In our patients, most cases were relapses after a prior ASCT, or they were not eligible for this procedure due to age or progression. However, all patients eligible for ASCT had an excellent outcome. In fact, the median PFS of patients consolidated with ASCT or allogeneic SCT was not reached, while in nonconsolidated patients it was 6 months $(P<0.001)$. Similarly, the need for response consolidation has also been observed after current promising new salvage therapies for relapsed HL, such as brentuximab vedotin, with a median PFS of 5.6 months. ${ }^{15}$ 


\section{Conclusion}

In conclusion, in our series of relapsed or refractory HL with a high rate of relapses after ASCT, the GemOx regimen showed good activity with manageable toxicity, low mobilization failure rates, and excellent outcomes when consolidated with hematopoietic stem cell transplantation. When frontline standard strategies fail, the GemOx regimen may be considered as another alternative for patients with relapsed or refractory HL, as in the case of new drugs, such as brentuximab vedotin or bendamustine.

\section{Acknowledgment}

We thank Mr Jonathan McFarland for his contribution in the revision of English language of the manuscript.

\section{Disclosure}

The authors report no conflicts of interest in this work.

\section{References}

1. The International Agency for Research on Cancer. WHO Classification of Tumours of Haematopoietic and Lymphoid Tissues. 4th ed. Swerdlow S, Campo E, Harris NL, et al, editors. Lyon, France: IARC; 2008.

2. Evens AM, Hutchings M, Diehl V. Treatment of Hodgkin lymphoma: the past, present, and future. Nat Clin Pract Oncol. 2008;5(9):543-556.

3. Younes A. CD30-targeted antibody therapy. Curr Opin Oncol. 2011;23(6):587-593.

4. Colpo A, Hochberg E, Chen YB. Current status of autologous stem cell transplantation in relapsed and refractory Hodgkin's lymphoma. Oncologist. 2012;17(1):80-90

5. López A, Gutiérrez A, Palacios A, et al. GEMOX-R regimen is a highly effective salvage regimen in patients with refractory/relapsing diffuse large-cell lymphoma: a phase II study. Eur J Haematol. 2008;80(2): $127-132$.

6. Rodríguez J, Gutierrez A, Palacios A, et al. Rituximab, gemcitabine and oxaliplatin: an effective regimen in patients with refractory and relapsing mantle cell lymphoma. Leuk Lymphoma. 2007;48(11):2172-2178.

7. Kuruvilla J, Nagy T, Pintilie M, Tsang R, Keating A, Crump M. Similar response rates and superior early progression-free survival with gemcitabine, dexamethasone, and cisplatin salvage therapy compared with carmustine, etoposide, cytarabine, and melphalan salvage therapy prior to autologous stem cell transplantation for recurrent or refractory Hodgkin lymphoma. Cancer. 2006;106(2):353-360.

8. Sibon D, Ertault M, Al Nawakil C, et al. Combined ifosfamide, etoposide and oxalipatin chemotherapy, a low-toxicity regimen for first-relapsed or refractory Hodgkin lymphoma after ABVD/EBVP: a prospective monocentre study on 34 patients. Br J Haematol. 2011;153(2):191-198.

9. Hasenclever D, Diehl V. A prognostic score for advanced Hodgkin's disease. International Prognostic Factors Project on Advanced Hodgkin's Disease. N Engl J Med. 1998;339(21):1506-1514.

10. Cheson BD, Horning SJ, Coiffier B, et al. Report of an international workshop to standardize response criteria for non-Hodgkin's lymphomas. NCI Sponsored International Working Group. J Clin Oncol. 1999;17(4):1244.
11. Kaplan EL, Meier P. Nonparametric estimation from incomplete observations. J Am Stat Assoc. 1958;53(282):457-481.

12. Peto R, Pike MC, Armitage P, et al. Design and analysis of randomized clinical trials requiring prolonged observation of each patient. II. analysis and examples. Br J Cancer. 1977;35(1):1-39.

13. Yang J, Shi Y, He X, et al. [Efficacy and safety evaluation of gemcitabine combined with oxaliplatin in lymphoma patients after failure of multiple chemotherapy regimens]. Zhonghua Zhong Liu Za Zhi. 2014;36(2): 137-140. Chinese.

14. Validire P, Fermé C, Brice P, et al. A multicenter study of gemcitabinecontaining regimen in relapsed or refractory Hodgkin's lymphoma patients. Anticancer Drugs. 2008;19(3):309-315.

15. Younes A, Gopal AK, Smith SE, et al. Results of a pivotal phase II study of brentuximab vedotin for patients with relapsed or refractory Hodgkin's lymphoma. J Clin Oncol. 2012;30(18):2183-2189.

16. Moskowitz AJ, Hamlin PA Jr, Perales MA, et al. Phase II study of bendamustine in relapsed and refractory Hodgkin lymphoma. J Clin Oncol. 2013;31(4):456-460.

17. Schmitz N, Pfistner B, Sextro M, et al; German Hodgkin's Lymphoma Study Group; Lymphoma Working Party of the European Group for Blood and Marrow Transplantation. Aggressive conventional chemotherapy compared with high-dose chemotherapy with autologous haemopoietic stem-cell transplantation for relapsed chemosensitive Hodgkin's disease: a randomised trial. Lancet. 2002;359(9323): 2065-2071.

18. Josting A, Rudolph C, Mapara M, et al. Cologne high-dose sequential chemotherapy in relapsed and refractory Hodgkin lymphoma: results of a large multicenter study of the German Hodgkin Lymphoma Study Group (GHSG). Ann Oncol. 2005;16(1):116-123.

19. Aparicio J, Segura A, Garcerá S, et al. ESHAP is an active regimen for relapsing Hodgkin's disease. Ann Oncol. 1999;10(5):593-595.

20. Moskowitz CH, Nimer SD, Zelenetz AD, et al. A 2-step comprehensive high-dose chemoradiotherapy second-line program for relapsed and refractory Hodgkin disease: analysis by intent to treat and development of a prognostic model. Blood. 2001;97(3):616-623.

21. Bartlett NL, Niedzwiecki D, Johnson JL, et al; Cancer Leukemia Group B. Gemcitabine, vinorelbine, and pegylated liposomal doxorubicin (GVD), a salvage regimen in relapsed Hodgkin's lymphoma: CALGB 59804. Ann Oncol. 2007;18(6):1071-1079.

22. Martín A, Fernández-Jiménez MC, Caballero MD, et al. Long-term follow-up in patients treated with Mini-BEAM as salvage therapy for relapsed or refractory Hodgkin's disease. Br J Haematol. 2001;113(1): 161-171.

23. Josting A, Kàtay I, Rueffer U, et al. Favorable outcome of patients with relapsed or refractory Hodgkin's disease treated with high-dose chemotherapy and stem cell rescue at the time of maximal response to conventional salvage therapy (Dex-BEAM). Ann Oncol. 1998;9(3): 289-295.

24. Rodriguez J, Rodriguez MA, Fayad L, et al. ASHAP: a regimen for cytoreduction of refractory or recurrent Hodgkin's disease. Blood. 1999;93(11):3632-3636.

25. Fermé C, Bastion Y, Lepage E, et al. The MINE regimen as intensive salvage chemotherapy for relapsed and refractory Hodgkin's disease. Ann Oncol. 1995;6(6):543-549.

26. Hertzberg MS, Crombie C, Benson W, Taper J, Gottlieb D, Bradstock KF. Outpatient fractionated ifosfamide, carboplatin and etoposide as salvage therapy in relapsed and refractory non-Hodgkin's and Hodgkin's lymphoma. Ann Oncol. 2006;17 Suppl 4:iv25-iv30.

27. Santoro A, Magagnoli M, Spina M, et al. Ifosfamide, gemcitabine, and vinorelbine: a new induction regimen for refractory and relapsed Hodgkin's lymphoma. Haematologica. 2007;92(1):35-41. 


\section{Publish your work in this journal}

OncoTargets and Therapy is an international, peer-reviewed, open access journal focusing on the pathological basis of all cancers, potential targets for therapy and treatment protocols employed to improve the management of cancer patients. The journal also focuses on the impact of management programs and new therapeutic agents and protocols on patient perspectives such as quality of life, adherence and satisfaction. The manuscript management system is completely online and includes a very quick and fair peer-review system, which is all easy to use. Visit http://www.dovepress.com/testimonials.php to read real quotes from published authors.

Submit your manuscript here: http://www.dovepress.com/oncotargets-and-therapy-journal 